\title{
Neuromodulation Methods to Suppress Tinnitus in Somatosensory Subtypes: A Case Series
}

\author{
Mark L. Johnson, Eugenia Bodenhamer-Davis, and Michael S. Gates \\ University of North Texas, Denton, Texas, USA
}

\begin{abstract}
Background: Some initial evidence suggests that neurofeedback and electrical stimulation therapy modalities may suppress tinnitus in some individuals. This study retroactively examined a case series of adults treated for tinnitus using varied neuromodulatory interventions, to explain relationships between etiological factors for tinnitus and differential responses to these interventions. Methods: Eight tinnitus client records were used to examine the efficacy of several different neuromodulation modalities used to treat tinnitus, which included neurofeedback, cranial electrotherapy stimulation (CES), and microcurrent electrical therapy (MET). Pre- and posttreatment measures (BAI, BDI, BHS, PSQI, THI, and TSS) were then compared for changes related to treatment outcomes. Results: Paired-sample $t$-tests showed the Tinnitus Severity Scale (TSS) and the Tinnitus Handicap Inventory (THI) to be significantly different following treatment, with tinnitus severity subsiding and sleep quality improving. Discriminant function analysis using the TSS, THI, and PSQI (Pittsburgh Sleep Quality Index) difference scores correctly classified all $(100 \%)$ participants in either the somatosensory or nonsomatosensory groups. Conclusions: Results of this small pilot study suggest that MET can improve tinnitus symptoms for individuals with a somatosensory form of the disorder in which tinnitus percept is unilateral or greater in degree on one side, fluctuates in intensity, and appears to involve musculoskeletal or central nervous system overarousal pathogenesis.
\end{abstract}

Keywords: tinnitus; microcurrent; Alpha-Stim

Citation: Johnson, M. L., Bodenhamer-Davis, E., \& Gates, M. S. (2016). Neuromodulation methods to suppress tinnitus in somatosensory subtypes: A case series. NeuroRegulation, 3(4), 150-161. http://dx.doi.org/10.15540/nr.3.4.150

*Address correspondence to: Mark Johnson, 627 Capitola Ave \#C, Edited by:

Capitola, CA 95010, USA. Email:markjohnson2@my.unt.edu

Rex L. Cannon, PhD, Neurogifted, Boynton Beach, Florida, USA

Copyright: ( 2016. Johnson et al. This is an Open Access article distributed under the terms of the Creative Commons Attribution License (CC-BY).

\section{Reviewed by:}

Rex L. Cannon, PhD, Neurogifted, Boynton Beach, Florida, USA Randall Lyle, PhD, Mount Mercy University, Cedar Rapids, lowa, USA

\section{Introduction/Background}

Tinnitus is a not a disease, but rather a vexing symptom in which a phantom sound is heard with no accompanying external source. Since tinnitus is a symptom that can be produced by numerous disorders and may have different causes and different pathophysiologies, searching for a single treatment for all forms of tinnitus could be futile (Møller, 1997). It has been suggested that the tinnitus percept may emerge from multiple, changing, overlapping subnetworks that can be affected by neuromodulatory activity (De Ridder et al., 2004). Thus, it has proven beneficial to identify individual functional subtypes of tinnitus that may respond to one or possibly a combination of treatments found to have some therapeutic efficacy.
Van de Heyning et al. (2007) described two major categories of tinnitus: objective tinnitus, caused by a real internal sound source inside the body and subjective tinnitus, an auditory phantom phenomenon caused by "a reorganization of the central auditory tract and auditory cortex, with a loss of suppression of neural activity" (p. 4). While Van de Heyning et al. give detailed causative variables for both objective and subjective forms of tinnitus, they suggest that subjective tinnitus primarily involves physiological, cochlear, phantom, central nervous system (CNS), and somatic variables.

Levine (2004) suggests that some subtypes of subjective tinnitus can result from multiple factors that synergistically produce the tinnitus symptoms. Factors such as pathological processes, certain classes of medications, psychosocial stress, sound 
exposure, or muscle activity related to head and neck maneuvers could all serve as "triggering factors" that can cause or can exacerbate symptomatic tinnitus.

\section{Somatic Tinnitus Model}

Clinical tinnitus has been linked to the somatosensory system (Levine, Abel, \& Cheng, 2003) and to limbic/thalamic involvement (Leaver et al., 2010; Mühlau et al., 2005). Aside from the auditory sensory system, only the somatosensory system has been shown to be closely related to tinnitus (Levine, Nam, Oron, \& Melcher, 2007). Human brain imaging studies have indicated that the neural generators for tinnitus might involve CNS as well as auditory and nonauditory pathways, in which movements and manipulations of certain upper extremities may increase (but usually not decrease) tinnitus severity (Simmons, Dambra, Lobarinas, Stocking, \& Salvi, 2008). The contribution of nonauditory centers in the pathogenesis and regulation of tinnitus is reinforced by studies showing that many patients have somatic tinnitus in which movements and manipulations of the eyes, head, neck, jaw, and shoulder can modulate the loudness and pitch of their tinnitus (Simmons et al., 2008). Levine suggests that variations in tinnitus perception may have somatic influences (i.e., increased severity upon waking may be due to nocturnal bruxism, or increased severity several hours into the day may be due to somatic movements reactivating the tinnitus severity; tinnitus upon waking from a nap may be due to poor head and neck posture while napping, etc.). It is possible that in some cases auditory or neurological pathogenesis combines with a triggering factor such as a somatic variable to onset tinnitus symptoms.

Levine and colleagues found that approximately $80 \%$ of their patients with tinnitus could transiently modulate their tinnitus with isometric head, neck, and jaw contractions. Levine (1999) purports that somatic modulation seems to be a fundamental attribute of tinnitus and that "somatic modulation may also account for reports of controlling tinnitus with physical methods such as acupuncture, manipulation, or scalp electrical stimulation" (p. 7). Levine et al. (2007) suggest there is evidence that the somatic tinnitus subgroup is most likely to respond to somatosensory-based treatment modalities. Some forms of tinnitus may lack this somatic component and be less likely to respond to physiological interventions.
Central Nervous System Sensitivity Model of Tinnitus

Patients with medically unexplained disorders or with symptoms such as tinnitus, that do not possess a manifest organic basis, are challenges for healthcare practitioners. Yunus (2009) was among the first to reconceptualize the traditional diseaseillness model and subsume symptomatically and diagnostically overlapping disorders under the unifying label of "central sensitivity disorders." According to Yunus, "central sensitivity syndromes (CSS) comprise a group of disorders that have overlapping clinical features, lack structural pathology, are based on neuroendocrine-immune dysfunction, and are bound by a common pathophysiological mechanism of central sensitization (CS). The common clinical features of these diseases include pain, fatigue, disturbed sleep, and hypersensitivity to various stimuli, including pain (e.g., pressure, heat, and electric) and environment (e.g., noise, stress, and chemicals). The Yunus criteria for categorizing symptoms as CSS involve two components: mutual associations among symptoms and evidence of CNS sensitivity among symptom clusters included in members. Both components need to be satisfied for inclusion in the CSS family (p. 400). Thus, CSS examples include irritable bowel syndrome (IBS), fibromyalgia (FMS), myofascial pain syndrome (MPS), headache/migraine, and temporomandibular disorder (TMD). While tinnitus is not yet considered a CSS condition, there are strong associations between tinnitus and other somatoform disorders. Hiller, Janca, and Burke (1997) reported the results of an international study conducted by the World Health Organization (WHO) that indicated tinnitus was more frequent among patients with somatization disorder $(42 \%)$ or hypochondriacal disorder $(27 \%)$. Tinnitus was also observed to occur in greater frequency than numerous other symptoms considered common to somatoform disorders. Tinnitus was associated with anxiety, depression, and with symptoms indicative of autonomic arousal. Headache $(46 \%)$, followed by rapid heart beating $(34 \%)$, chest pain $(29 \%)$, back pain $(24 \%)$, and abdominal pain $(22 \%)$ had the highest associations with tinnitus. Hiller et al. concluded that idiopathic tinnitus may be a somatoform symptom, that there may be substantial comorbidity among tinnitus and overlapping conditions, and that common mechanisms of arousal and somatic anxiety may link tinnitus with other forms of somatization, such as temporomandibular disorder (TMD). 
Van de Heyning et al. indicated that "somatosensory modulation of the auditory system can be caused by influences emanating from, in particular, the jaw and masticatory muscles and from the neck" (p. 5). Wright and Bifano (1997) reported a much higher incidence of tinnitus among patients with temporomandibular disorder (TMD) compared to age-matched controls. They also found that TMD therapy improved tinnitus in $46-96 \%$ of their patients who had these coexisting symptoms.

Relationships Between Central Nervous System Overarousal, Chronic Pain, and Tinnitus

Given the strong association between somatization and tinnitus, it seems relevant to consider the relationship between pain and tinnitus. Similarities between tinnitus and phantom (missing limb) pain have brought attention to the role that the CNS may play in these conditions (De Ridder, Elgoyhen, Romo, \& Langguth, 2011; Saunders, 2007). Møller (1997) compared chronic pain mechanisms with that of tinnitus and concluded there is strong evidence that chronic pain and certain types of tinnitus are similar in that each appears to have a peripheral etiology but may actually be caused by central nervous system pathophysiology.

Hiller et al. (1997) hypothesized that tinnitus and somatization might be linked through common mechanisms of arousal and somatic anxiety (p. 613). Sleep bruxism has been construed as an oromotor manifestation of microarousal in which anxiety is an underlying component. Bracha, Ralston, Williams, Yamashita, and Bracha (2005) suggest that clenching-grinding spectrum behaviors be construed in a neuroevolutionary perspective as fear-circuitry disorders linked to the activation of fear circuits and anxiety and that clenching-grinding, sleep bruxism, myofascial pain, craniomaxillofacial pain, musculoskeletal pain, temporomandibular disorders, crofacial pain, sleep bruxism, fibromyalgia/chronic fatigue spectrum disorders are all linked-possibly all or most indicating persistent fear-circuitry activation. These researchers noted the risk factors for sleep bruxism, which has been associated with increased tinnitus severity, include anxiety, a highly stressful life, excessive caffeine consumption, hypnopompic/hypnagogic imagery, and various sleep disorders. They also noted that prolonged jaw clenching has been clinically observed among developmentally disabled populations during episodes of fear or rage. Suggestive of the role of anxiety and stress in tinnitus as well as the possible effectiveness of addressing physiological or somatosensory variables in treatment of tinnitus is the fact that Nakai et al. (2008) reported the successful treatment of a deaf patient's tinnitus using an autogenic relaxation training method.

Treatment Response of Somatosensory
Subgroup of Tinnitus Levine et al. (2007) identified tinnitus subgroups that may respond well to somatosensory-based treatment modalities. "Somatosensory tinnitus" refers to tinnitus that can be activated through disorders of the upper cervical region and head. They summarized studies that included cervical manipulation, acupuncture, trigger point injections, TMD therapy, and electrical stimulation of the scalp and auricle. They reported that the characteristics of responders to cervical manipulation are largely unknown. Individuals likely to respond to acupuncture were characterized by symmetric hearing and tinnitus lateralized mainly to one side. This combination of symmetrical hearing and unilateral tinnitus suggests it is unlikely this condition is solely auditory in origin. An intervention using trigger point injections at cervical and jaw muscle locations transiently abolished tinnitus. This was found more often for patients with higher cervical tension on their tinnitus side. Wright and Bifano (1997) found that many times TMD therapy improved or resolved tinnitus symptoms in patients with coexisting TMD and tinnitus and developed a list of questions that could help practitioners determine which patients were likely to benefit from this approach. Most patients who responded positively to this treatment had normal hearing and their tinnitus was ipsilateral to their TMD. Levine also summarized three studies using electrical stimulation of the scalp and auricle in which the best responders shared characteristics of symmetric hearing and unilateral tinnitus, again suggesting somatically-induced tinnitus.

Neurophysiological and Electrophysiological Models of Tinnitus

Saunders (2007) noted that a neurophysiological model of tinnitus accredited to Jastreboff (1990) considers additional central nervous system (CNS) contributions. Many cases of tinnitus occur with hearing loss due to intense sound exposure, aging factors, or from exposure to certain drug agents (salicylates, aminoglycoside antibiotics, quinine, or cisplatin; König, Schaette, Kempter, \& Gross, 2006; Sindhusake, et al., 2004). Anatomic alterations due to peripheral injury may result in maladaptive neuroplastic alterations. Saunders suggests that tinnitus may be the result of maladaptive alterations in brain neuroplasticity: "One theory proposes that these changes upset the balance between excitatory 
and inhibitory brain processes with the result being neural hyperactivity" (p. 314).

A recent study showed that tinnitus is much more frequently reported in patients who are electromagnetically hypersensitive when compared to controls (Landgrebe, Frick, Hauser, Hajak, \& Langguth, 2009); and Vernon (1987) summarized attempts, primarily in the 1980s, to employ different types of electrical administration at a variety of cranial locations to suppress tinnitus. Many of these attempts involved surgically implanted electrodes. There have also been a small number of studies using noninvasive methods to apply electrical stimulation to treat tinnitus, one of which involved a transcutaneous headband device that had a small degree of success (Dobie, Hoberg, \& Rees, 1986).

Saunders states that tinnitus should be viewed as a complex constellation of neural changes in which no one brain location is implicated, and this may explain why it is not amenable to any single treatment. In fact, studies have identified a number of specific alterations to brain regions and brain wave patterns during active tinnitus. Dohrmann, Weisz, Schlee, Hartman, and Elbert (2008) proposed that at least three neural network regions (temporal, frontal, and limbic) are involved in tinnitus. Citing electroencephalographic (EEG) studies, they noted that individuals with tinnitus abnormalities tend to exhibit increased delta band activity in the $1.5-4 \mathrm{~Hz}$ frequency range and reduced alpha band activity in the $8-12 \mathrm{~Hz}$ range. Weisz, Moratti, Meinzer, Dohrmann, and Elbert (2005) found this abnormal activity to be especially pronounced in right temporal and left frontal areas. Dohrmann et al. applied neurofeedback to 21 subjects (for ten 30-min sessions at frontal sites (C3, C4, FC1, and FC2) and found that patients who successfully modified their brain activity at these sites had the greatest reductions in their tinnitus. However, only the patients who successfully modified both alpha and delta frequency bands achieved the strongest relief.

Likewise, Kahlbrock and Weisz (2008) found that patients who were able to normalize their brain activity patterns achieved significant reductions in their tinnitus severity. Furthermore, significant reduction of delta $(1.3-4.0 \mathrm{~Hz})$ frequency band power was observed in temporal regions while residual inhibition occurred. "Delta activity is a characteristic oscillatory activity generated by deafferented/deprived neuronal networks. This implies that RI (residual inhibition) effects might reflect the transient reestablishment of balance between excitatory and inhibitory neuronal assemblies" (p. 1). These researchers concluded that the ability to decrease delta frequency amplitude and increase alpha frequency amplitude in temporal regions predicted lowered tinnitus volume.

Weiler, Brill, Tachiki, and Schneider (2002) demonstrated that brainwave biofeedback (neurofeedback) successfully disrupted a woman's chronic bilateral tinnitus to a point where it occurred only occasionally. Treatment reduced delta and theta power and increased alpha band power. The success of this brainwave modification approach suggests that other neuromodulation methods might also be effective with tinnitus.

Cranial Electrotherapy Stimulation
Microcurrent Electrical Therapy (MET)
The cranial electrotherapy stimulation
microcurrent electrical therapy (MET)
$\begin{array}{ll}\text { brain }\end{array}$ stimulation devices have been used to treat anxiety, depression, sleep, and pain. Mercola and Kirsch (1995) explain that "MET works because of its ability to stimulate cellular physiology and growth [and] correct application of MET to an injured site augments the endogenous current flow, allowing cells in the traumatized area to regain their capacitance. Resistance is reduced, thereby allowing bioelectricity to flow through and reestablish homeostasis. This process helps to initiate and perpetuate the many biochemical reactions that occur in healing" (p. 110). In an application of this MET mechanism, McMakin (1998), noting that "repeated experience of a sympathetic stress response will cause predictable tissue changes leading to tightening of the myofascia and muscle contracture and promoting formation of a trigger point" (p. 30), used microcurrent therapy delivered through graphite/vinyl gloves to significantly reduce myofascial pain in four of five chronic cervical pain patients.

In a 2006 unpublished dissertation, Richard Kennerly used quantitative electroencephalography (qEEG) to measure the effect of cranial electrotherapy stimulation (CES) on brainwave activity. He found that a single 30-min administration of CES treatment with the Alpha-Stim AID Cranial Electrotherapy Stimulator unit (Electromedical Products International) produced an increase in alpha relative power along with a concomitant decrease in both delta and beta relative power. These changes in pre- and post-qEEG relative power bands were consistent with the effects of CES reported in the literature, such as increased relaxation and decreased anxiety. These 
results also involved the same frequency bands targeted in the neurofeedback studies reported above that resulted in tinnitus improvements.

Engelberg and Bauer (1985) reported using the Alpha Stim 2000 microcurrent electrotherapy (MET) instrument to treat tinnitus and obtained symptom improvement in $82 \%$ of 33 ears of 20 subjects. Subjects received 1 to 17 treatments to the outer ear, with most receiving just one or two treatments. In most cases, electrical stimulation administered to 13 specific auricle points ipsilateral to the tinnitus resulted in amelioration of the tinnitus. The lasting effects of the improvements ranged from 20 minutes to 6 months. Most subjects reported the improvement lasted 3 days or less. The procedure was applied with the subject holding a ground in one hand ipsilateral to the tinnitus while the therapist stimulated a circular pattern of aurical points on the outer ear with a single microcurrent probe

The research cited above appears to provide strong support for neurophysiological and electrophysiological explanatory models of tinnitus as well as the involvement of CNS/sympathetic overarousal mechanisms. The preliminary research cited has demonstrated good success using electrotherapy stimulation (CES and MET) devices such as the Alpha-Stim instrument to affect the particular brainwave frequencies involved in tinnitus. In addition, these devices have proven effective for CNS overarousal and pain disorders, suggesting that CES and MET hold promise for the treatment of tinnitus, especially the somatosensory subtype.

\section{Somatosensory Subtype}

An ongoing review of relevant literature led to speculation that the tinnitus somatosensory subtype would be most likely to respond to treatment. The somatosensory subtype profile included three components: 1) asymmetric tinnitus (i.e. more unilateral than bilateral), 2) fluctuations in tinnitus percept (i.e. volume/intensity fluctuates as opposed to remaining stable and consistent), and 3) tinnitus changes can be influenced by head/neck neuromuscular activity. The following study was an effort to provide additional data to test this clinical hypothesis.

\section{Methods}

\section{Participants}

Subjects for this study were eight individuals who completed neurofeedback, CES, or MET as part of their treatment at a university-based neurotherapy clinic or at a private mental health clinic. In addition to the primary symptoms for which four subjects sought treatment, the other four subjects all sought treatment for unilateral or bilateral tinnitus. Table 1 shows the demographic characteristics of the subject group. Participants included seven Caucasian females and one male with ages ranging from 27 to 70 . Most had experienced tinnitus symptoms for several years. Data for these subjects was collected from 2009-2010 archived records of the university clinic and 2012-2013 archived records from the private mental health clinic.

As a prerequisite to treatment, clients whose records were used in this study were required to have read and signed an informed consent document that explained the neurotherapy treatment requirements and possible side effects or risks of the treatments. Clients also gave written permission for any information gained through their treatment to be used anonymously for educational or research purposes. Each client provided medical and family history and completed intake assessments. History included screening for pregnancy and demand-type cardiac pacemakers, that are contraindications for treatment with electrical stimulation (See Table 1).

All subjects were assessed pre- and posttreatment using the following psychometric instruments: Tinnitus Severity Scale (TSS), Tinnitus Handicap Inventory (THI), and Pittsburgh Sleep Quality Index (PSQI). Tinnitus subtype classification was based on subjective self-report prior to treatment. Five of the eight subjects also completed BAI, BDI, and BHS measures (See Table 3). However, these measures were not included in the statistical analysis. 


\section{Table 1}

Demographic Summary

\begin{tabular}{|c|c|c|c|c|c|c|c|c|}
\hline $\begin{array}{l}\text { Case } \\
(N=8)\end{array}$ & Age & Gender & Ethn $^{a}$ & Side & Length $^{b}$ & Fluc $^{c}$ & Musc $^{d}$ & Improved? \\
\hline 1 & 56 & $F$ & C & Left & 6 years & Yes & Yes & Yes \\
\hline 2 & 61 & $F$ & C & Left/Bi & 2 years & Yes & Yes & Yes \\
\hline 3 & 27 & $F$ & $\mathrm{C}$ & Same & 4 years & Yes & No & No \\
\hline 4 & 60 & $F$ & $\mathrm{C}$ & Same & 3 years & Yes & No & No \\
\hline 5 & 70 & $F$ & C & Same & 30 years & No & No & No \\
\hline 6 & 39 & $F$ & C & $\mathrm{Bi}$ & 1 year & Yes & Yes & Yes \\
\hline 7 & 25 & $F$ & $\mathrm{C}$ & Uni & 2 years & Yes & Yes & Yes \\
\hline 8 & 32 & $M$ & $\mathrm{C}$ & $\mathrm{Bi}$ & 5 years & Yes & Yes & Yes \\
\hline
\end{tabular}

\section{Instrumentation}

\section{Neurofeed back Instrumentation and Protocol} Brainwave training in the form of EEG biofeedback or neurofeedback was provided using BrainMaster Atlantis version 3.0 software $(n=1)$. BrainMaster equipment was set to include a $60-\mathrm{Hz}$ notch filter, 256 data-sampling rate, $125 \mu \mathrm{v}$ artifact threshold, and peak-to-peak amplitude scale. This equipment was set to inhibit delta $(1.5-4.0 \mathrm{~Hz})$ and high beta $(20-30 \mathrm{~Hz})$ and to reward alpha $(8-12 \mathrm{~Hz})$. Active electrode placements were guided by qEEG to target the signature tinnitus activity in the delta and alpha ranges. The left (A1) and right (A2) ears were used for reference and ground sites.

\section{CES and MET Device}

[Electromedical Products International, Inc.] AlphaStim 100 combined microcurrent and cranial electrotherapy stimulators were used. These battery-powered devices generate current at 0 to 500 microamperes continuously adjustable. The frequency is $0.5 \mathrm{~Hz}$ (pulses per second) combined with a constant $0.4 \mathrm{~Hz}$. The average pulse rate is $0.8 \mathrm{~Hz}$. Pulse widths vary between $0.25,0.5,0.75$, and $1 \mathrm{~s}$. At $500 \mu \mathrm{A}$ the charge per pulse varies between 125, 250, 375, and 500 microcoulombs $(\mu \mathrm{C})$. Every $10 \mathrm{~s}$ the total charge is 1.25 millicoulombs $(\mathrm{mC})$ in each direction. The impedance ranges within which the waveform parameters remain valid are from $100 \Omega$ to $10 \mathrm{~K} \Omega$. The waveform is composed of bipolar asymmetric rectangular waves at a $50 \%$ duty cycle repeating periodically at 10-s intervals. The waveform is balanced to achieve 0 net current in either direction. Current alternated (10 s on, alternating with $2 \mathrm{~s}$ off) when using the probe function, which applies current to sites using two electrically conducting, manually administered probes. When treatment included the use of the microelectric current therapy using the probes, clients were asked to provide a subjective rating of their tinnitus severity, based on a scale of 0 to 10 ( 0 = percept inaudible; $1=$ barely noticeable; $10=$ intolerable), following each auricle administration of the current (See Figure 1). 

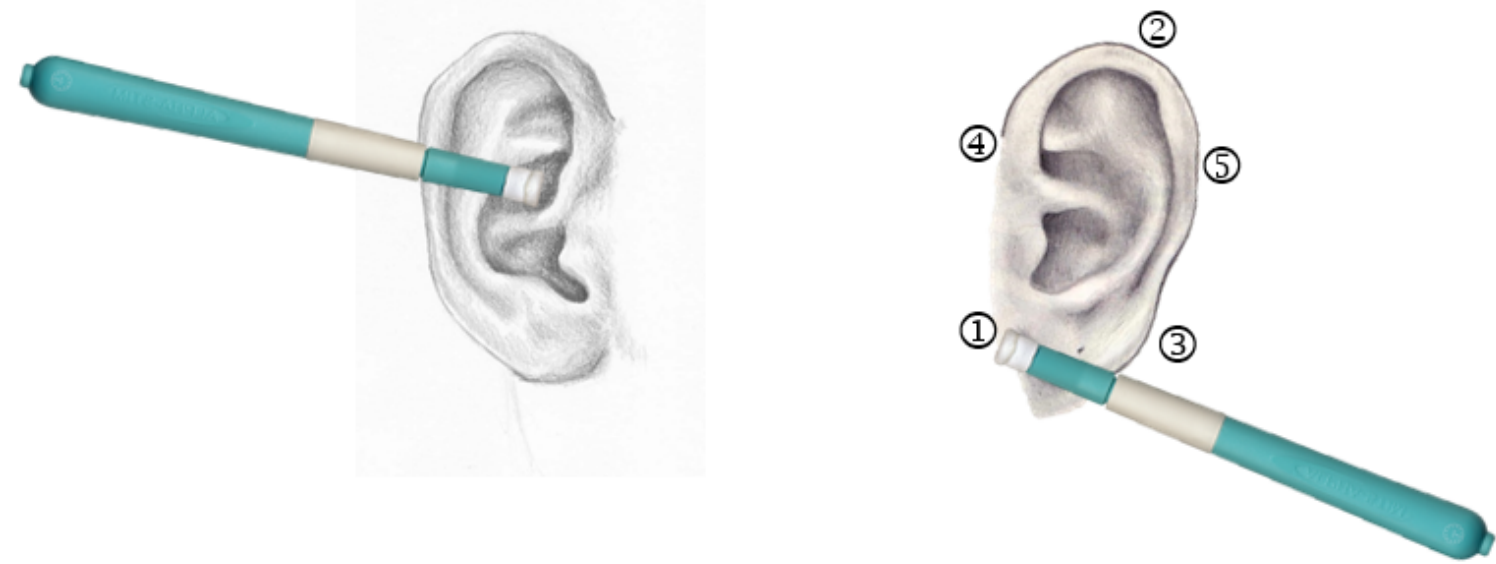

Step 1: Insert probe in concha cymba or concha cavum of left ear (not in canal).

Step 2: Treat in 10-s intervals at locations 1-5 in star-shaped pattern of contralateral ear.

Step 3: Switch ears and repeat this procedure (i.e. 1 round of treatment).

Step 4: Repeat process while monitoring symptom reduction or until treatment plateau occurs.

*Our study averaged approximately 6 rounds (with breaks) for approximately 20-30 minutes of treatment.

Figure 1. Tinnitus MET Protocol.

\section{Case 1}

A 56-year-old female developed constant severe unilateral, left-side tinnitus immediately following a hysterectomy surgical procedure. Her tinnitus had persisted for the past 5 years and fit the somatosensory subtype profile. The client was treated with qEEG-based neurofeedback at T4 with a 2-9 Hz and $20-30 \mathrm{~Hz}$ inhibit protocol. On the eighth day of training at this site, the client reported her tinnitus had disrupted for a full day for the first time in years. Training was continued at temporal/parietal (T4/T6/P4) sites, which continued to produce a fluctuation in her tinnitus between sessions (and, at times, modulate pitch or intensity within sessions). By session 14, the client reported that her tinnitus was suppressed $50 \%$ of the time (either completely on, or completely off). The client's baseline qEEG indicated the highest delta amplitude and lowest alpha amplitude at site F3. Additional gains were reported by the client after receiving neurofeedback treatment at this site. She reported that the tone and severity of the tinnitus improved, and usually within an hour following each session her tinnitus would attenuate and remain suppressed $75 \%$ of the time thereafter.

Given that the Alpha-Stim 100 has been shown to produce a similar increase in alpha while decreasing delta and high beta (Kennerly, 2006), it was hypothesized that adding CES to the treatment regimen might augment clinical results. The client used the CES component of the Alpha-Stim 100 (attached by ear lobe clips) for $>20$ sessions at home. She reported that using the CES device seemed to keep her tinnitus subdued and better managed when not receiving tinnitus NF training. The therapist then speculated that treating auricle sites on the outer ear that may have more precise correlation with tinnitus might be more helpful, such as in the case of treating pain with MET at the physical location of the pain sensation. The therapist then administered the microcurrent electrical therapy component of the Alpha-Stim 100 using the probes, in the same manner as would be used with this MET instrument for a pain protocol. A succession of repeated administrations of MET at five points (in a star-shaped pattern) around auricle with a simultaneous probe in a centered location of the opposite outer ear (not in the canal) gradually suppressed the active tinnitus into full remission within the first treatment administration. The client then self-administered this protocol at home and found the tinnitus to be suppressed $80-90 \%$ of the time and found it to be otherwise barely audible. However, the client noted that when she stopped using the Alpha-Stim/MET for periods of time, her tinnitus returned within "several days." Client's resuming use of the device would reinstate tinnitus suppression (subjective rating from an 8 to a 3 on a scale of 0 to 10$)$.

\section{Case 2}

A 61-year-old female developed bilateral tinnitus with spontaneous onset 2 years prior to her clinical treatment. This client also met criteria for somatosensory subtype, having significant muscle 
tension in shoulder and neck areas. She was initially treated with the CES component of the Alpha-Stim 100 (attached by ear lobe clips) for 20 sessions with no change in tinnitus symptoms. However, a single treatment session using the MET modality of the Alpha-Stim 100 resulted in nearly complete suppression of the tinnitus. The same pain protocol consisting of a succession of repeated administrations at five points in and around each auricle (first on her left ear, and then eventually on the right ear) gradually reduced the tinnitus to nearly full remission (subjective rating from a 5 to a 0.5 on a scale of 0 to 10). Her tinnitus had not returned at a 1-month follow-up. Interestingly, the client also reported modulation of her tinnitus when the probe was placed on her upper trapezius/neck muscle (a divergence to address upper back/neck tension), adding further support to the musculoskeletal involvement in this subtype.

\section{Case 3}

A 27-year-old female developed bilateral tinnitus with spontaneous onset 5 years prior to initiating treatment. She did not meet the usual criteria for a somatosensory subtype and had a complex history and clinical presentation. This client had received audiological testing and learned she had nerve damage and hearing loss. She also reported a history of TMD. Her tinnitus was severe and consisted of three different pitches bilaterally. She was initially treated with CES, but discontinued after six sessions due to headaches, dizziness, and fatigue. MET was then tried, but discontinued after six sessions when client reported an increase in tinnitus pitch loudness. The loudness returned to its previous level 1 to 2 weeks later (subjective rating from a 10 to a 10 on a scale of 0 to 10 ).

\section{Case 4}

A 60-year-old female developed bilateral tinnitus with spontaneous onset 3 years prior to entering treatment. She reported a history of TMD and did not meet criteria for somatosensory subtype. She received 20 CES and 20 MET treatments with no reported improvement from either modality (subjective rating from a 4 to a 6 on a scale of 0 to 10).

\section{Case 5}

A 70-year-old female had developed bilateral tinnitus with spontaneous onset 30 years prior. She did not meet criteria for somatosensory subtype. She received 20 CES and 20 MET treatments with no reported change from either modality (subjective rating from a 3 to a 2 on a scale of 0 to 10).
Case 6

A 39-year-old female developed bilateral tinnitus following a postcraniotomy for left-frontal meningioma that was resected 1 year prior to her treatment. The resection resulted in the client experiencing constant headaches and a continuous "clicking sound." She fit the somatosensory subtype profile and was treated with MET. Her tinnitus almost fully remitted in a single session (subjective rating from an 8 to a 1 on a scale of 0 to 10). At a 1.4-year follow-up, her tinnitus remained in remission.

\section{Case 7}

A 25-year-old female developed unilateral tinnitus with an onset that occurred 2 years prior to her clinical treatment. Her tinnitus may have resulted from an automobile collision after which she experienced onset of frequent headaches (1-3 times per week) and persistent tinnitus in the form of a "whooshing sound" that progressively worsened, especially over the 4 months prior to treatment. The subject noted that her tinnitus was unilateral, in the left ear, and fluctuated both with movement and body position (i.e. her tinnitus worsened when changing from standing to seated or lying down positions). She met criteria for somatosensory subtype. She received an initial CES treatment for headache (using ear clip locations) that resulted in remission of both her headache pain and her tinnitus (subjective rating from a 6 to a 0 on a scale of 0 to 10). At a 1.7-year follow-up, her tinnitus remained in remission.

Case 8

A 32-year-old male reported being diagnosed with a hemangioblastoma that was resected in February 2007. He reported that he had radiation, but eventually the tumor returned and metastasized and spread to bone and other areas. After exhaustive treatment, he eventually went into remission of the cancer, but reported a subsequent history of tinnitus that met criteria for somatosensory subtype. His tinnitus was suppressed to full remission following a single treatment (subjective rating from a 6 to a 0 on a scale of 0 to 10). At a 1-year follow-up, he reported his tinnitus had remained fully suppressed for 3 months, then resumed, but at a lesser and more tolerable level. He did not receive follow-up treatment because his cancer had returned, and he was involved in additional treatment for that condition. 


\section{Analysis of Results}

Paired $t$-tests were performed to determine if the difference scores between the pre- and posttests for the TSS, THI, and PSQI were statistically significant. In addition, discriminant function analyses (DFA) were completed to determine the efficacy of using the difference scores on the TSS, THI, and PSQI to classify participants in either the somatosensory or nonsomatosensory groups. DFA also was used to predict whether participants reported that tinnitus symptoms were better or worse after exposure to NF, CES, or MET treatments. Because only one participant received NF treatments before exposure to CES and MET, NF was excluded as a predictor in the second discriminant analysis. DFA normally uses continuous variables to predict membership in two or more mutually exclusive groups, which was the case in the first analysis. However, the second DFA used categorical variables to predict the likelihood that participants indicated their tinnitus symptoms improved after each treatment. Both paired sample $t$-tests and DFA require exogenous variables that are normally distributed, but each analysis is fairly robust to violations to the underlying assumptions during each test. The results below were validated using nonparametric bootstrapping methods, which produced nearly identical results for both the paired $t$-tests and each DFA. However, since the sample size in this exploratory study consisted of only eight individuals, which by itself limits the generalizability of the findings, only the parametric results for each analysis conducted will be described.

Paired-sample $t$-tests showed the scores for the Tinnitus Severity Scale (TSS), $t_{(d t=7)}=-2.94$, $p=.022$, and the PSQI, $t_{(d f=7)}=-2.53, p=.039$, were statistically different following treatment. The posttreatment Tinnitus Handicap Inventory (THI), $t_{(d f=7)}=-1.94, p=.047$, one-tailed, had a statistically significant result from pretreatment, indicating that tinnitus handicap indicators subsided after neuromodulation treatment. The difference scores from the pre- and posttests on the TSS, THI, and $P S Q \mid$ were then used to classify participants into one of two groups, somatosensory and nonsomatosensory. With only two groups, DFA yields only one discriminant function. The first discriminant analysis using difference scores had an Eigenvalue of 9.56 that accounted for $100 \%$ variance, with a canonical correlation of .952. The canonical correlation when squared is the proportion of the total variability explained by differences between groups. Stated differently, a canonical correlation near 1 suggests that the function discriminates well (1.00 is perfect). The analysis had a Wilks' $\lambda=.094, X_{(d f=3)}^{2}=10.62, p=.014$. The calculated $\eta^{2}$ is $1-\lambda=.91$, indicating that $91 \%$ of the variance in the categorical grouping variable is shared with the linear combination of the TSS, THI, and PSQI difference scores. A chi-square transformation of Wilks lambda was used to determine significance of Wilks' $\lambda$, and $p$-values smaller than .10 are significant, indicating that the group means differ. Similarly, the high chi-square, 10.6, indicated the difference scores on the three measures discriminate well between somatosensory and nonsomatosensory participants. Table 4 presents the classification table for the DFA predicting membership in either the somatosensory or nonsomatosensory groups. By using the difference scores from the TSS, THI, and PSQI, the discriminant function correctly classified all participants and supports a tentative conclusion from this study that the neuromodulation methods of neurofeedback, CES and MET had a differential impact on symptom reports of subjects having the somatosensory subtype of tinnitus.

Categorical variables representing whether participants were classified as somatosensory or nonsomatosensory and received either CES or MET were used to predict if participants reported better or worsening tinnitus symptoms after each individual treatment during the study. There were $N=149$ subjective measures of whether tinnitus symptoms improved, one following each respective treatment (including in-session trials of MET). The second discriminant function analysis had an Eigenvalue of 1.327 that accounted for $100 \%$ variance with a canonical correlation of .755 . Wilks' $\lambda=.43$, $X_{(d f=3)}^{2}=122.89, p<.001$. The calculated $\eta^{2}$ is $1-$ $\lambda=.57$, indicating that $57 \%$ of the variance in the categorical grouping variable is shared with the linear combination for the somatosensory, CES, and MET categorical variables. The $X^{2}$ of 122.9 was significant and its value suggests these variables discriminate well between those who reported subsiding symptoms and those who did not. Table 5 presents the classification table for the DFA, predicting membership in either the "better or notbetter" groups. The categorical variables indicating whether individuals were somatosensory and received CES and/or MET correctly classified $94.6 \%$ of the subjective responses recorded after each neuromodulation treatment. In other words, knowing whether a participant was somatosensory or not, together with the type of treatment received, strongly supports the contention that neuromodulation methods, especially MET, can substantively reduce tinnitus symptoms after each treatment. These 
findings are consistent with those obtained in the much earlier study done by Engelberg and Bauer
(1985) who used the Alpha-Stim 2000, a large (MET) clinical device.

\section{Table 2}

Tinnitus Severity, Handicap, and Sleep Inventory Pre and Post Scores

\begin{tabular}{|c|c|c|c|c|c|c|}
\hline \multirow{2}{*}{$\begin{array}{l}\text { Case } \\
(N=8)\end{array}$} & \multicolumn{2}{|c|}{$\mathrm{TSS}^{\mathrm{a}}$} & \multicolumn{2}{|c|}{$\underline{\mathrm{THI}}^{\mathrm{b}}$} & \multicolumn{2}{|c|}{$\underline{\mathrm{PSQI}^{\mathrm{C}}}$} \\
\hline & Pre & Post & Pre & Post & Pre & Post \\
\hline 1 & 8 & 3 & 60 & 34 & 13 & 7 \\
\hline 2 & 5 & 0.5 & 20 & 12 & 5 & 5 \\
\hline 3 & 10 & 10 & 64 & 62 & 23 & 2 \\
\hline 4 & 4 & 6 & 6 & 4 & 3 & 2 \\
\hline 5 & 3 & 2 & 4 & 8 & 2 & 3 \\
\hline 6 & 8 & 1 & 100 & 4 & 18 & 6 \\
\hline 7 & 6 & 0 & 40 & 6 & 13 & 7 \\
\hline 8 & 6 & 0 & 28 & 14 & 14 & 7 \\
\hline
\end{tabular}

Note. ${ }^{a}$ Tinnitus Severity Scale. ${ }^{b}$ Tinnitus Handicap Inventory. ${ }^{c}$ Pittsburgh Sleep Quality Index.

\section{Table 3}

Pre- and Posttreatment Scale Scores

\begin{tabular}{|c|c|c|c|c|c|c|}
\hline \multirow{2}{*}{$\begin{array}{l}\text { Case } \\
(N=8)\end{array}$} & \multicolumn{2}{|c|}{$\underline{B A I^{a}}$} & \multicolumn{2}{|c|}{$\mathrm{BDI}^{\mathrm{b}}$} & \multicolumn{2}{|c|}{$\mathrm{BHS}^{\mathrm{c}}$} \\
\hline & Pre & Post & Pre & Post & Pre & Post \\
\hline 1 & 36 & 10 & 23 & 5 & 10 & 4 \\
\hline 2 & 4 & 1 & 0 & 1 & 1 & 1 \\
\hline 3 & 16 & 5 & 7 & 3 & 2 & 1 \\
\hline 4 & 0 & 2 & 2 & 1 & 9 & 1 \\
\hline 5 & 4 & 3 & 1 & 6 & 4 & 2 \\
\hline 6 & N/A & $\mathrm{N} / \mathrm{A}$ & $\mathrm{N} / \mathrm{A}$ & N/A & $\mathrm{N} / \mathrm{A}$ & N/A \\
\hline 7 & N/A & $N / A$ & $\mathrm{~N} / \mathrm{A}$ & $\mathrm{N} / \mathrm{A}$ & $\mathrm{N} / \mathrm{A}$ & N/A \\
\hline 8 & $\mathrm{~N} / \mathrm{A}$ & $\mathrm{N} / \mathrm{A}$ & $\mathrm{N} / \mathrm{A}$ & N/A & $\mathrm{N} / \mathrm{A}$ & $\mathrm{N} / \mathrm{A}$ \\
\hline
\end{tabular}

Note. ${ }^{a} B A I-B e c k$ Anxiety Inventory. ${ }^{b} B D I-B e c k$ Depression Inventory. ${ }^{c} B H S$ - Beck Hopelessness Scale. 


\section{Table 4}

Discriminant Function Analysis Somatosensory Classification Results

\begin{tabular}{|c|c|c|c|c|}
\hline \multirow[b]{2}{*}{ Somatosensory } & & \multicolumn{3}{|c|}{ Predicted Group Membership } \\
\hline & & NS & $S$ & Total \\
\hline \multirow{4}{*}{ Original } & Nonsomatosensory & 3 & 0 & 3 \\
\hline & Somatosensory & 0 & 5 & 5 \\
\hline & Nonsomatosensory & 100.0 & 0.0 & $100.0^{\mathrm{a}}$ \\
\hline & Somatosensory & 0.0 & 100.0 & $100.0^{\mathrm{a}}$ \\
\hline
\end{tabular}

Note. ${ }^{a} 100.0 \%$ of original grouped cases correctly classified.

\section{Table 5}

Discriminant Function Analysis Better/Not-Better Classification Results

\begin{tabular}{lcrccc}
\hline \multicolumn{1}{l}{ Better } & & \multicolumn{2}{c}{ Predicted Group Membership } \\
\hline \multirow{2}{*}{ Original } & Count & Not better & Not better & Better & Total \\
& & Better & 104 & 7 & 111 \\
& $\%$ & Not better & 93.7 & 37 & 38 \\
\end{tabular}

Note. ${ }^{a} 94.6 \%$ of original grouped cases correctly classified.

\section{Conclusions}

Microcurrent electrical therapy (MET) at sequential auricle sites appears to provide a method of reorganizing and re-establishing neural homeostasis at the intercept of the involved somatic and auditory pathways of somatosensory tinnitus. While neurofeedback has shown some possibility of tinnitus interruption, and CES has also demonstrated limited success, the specificity of MET at particular auricle sites seems to show the most promise and the most efficiency. Results of this clinical case series suggests that the somatosensory subgroup of tinnitus sufferers respond better to these neuromodulation treatments than other tinnitus subgroups. This somatosensory subgroup is characterized by nonbilateral tinnitus pitch, pitch that may fluctuate in tone/intensity, and EMG modulation of pitch. Further, both CES and MET appeared to reduce symptoms for postcraniotomy ("clicking" or "wooshing" sounds) and/or TBI-induced tinnitus. Even though this study was limited by such a small number of participants, the somatosensory subgroup responded to the MET treatment in single sessions, indicating that it, as well as CES, are promising treatments for tinnitus that warrant further exploration.

\section{References}

Bracha, H. S., Ralston, T. C., Williams, A. E., Yamashita, J. M., \& Bracha, A. S. (2005). The clenching-grinding spectrum and fear circuitry disorders: clinical insights from the neuroscience/paleoanthropology interface. CNS Spectrums, 10(4), 311-318. /s109285290002263x https://dx.doi.org/10.1017

De Ridder, D., Elgoyhen, A. B., Romo, R., \& Langguth, B. (2011). Phantom percepts: Tinnitus and pain as persisting aversive memory networks. Proceedings of the National Academy of Sciences of the United States of America, 108(20), 80758080. https://dx.doi.org/10.1073/pnas.1018466108

De Ridder, D., Vanneste, S., Weisz, N., Londero, A., Schlee, W., Elgoyhen, A. B., \& Langguth, B. (2004). An integrative model of auditory phantom perception: Tinnitus as a unified percept of interacting separable subnetworks. Neuroscience \& Biobehavioral Reviews, 44, 16-32. https://dx.doi.org/10.1016 /j.neubiorev.2013.03.021

Dobie, R. A., Hoberg, K., E., \& Rees, T. S. (1986). Electrical tinnitus suppression: A double-blind crossover study. Otolaryngology - Head and Neck Surgery, 95(31), 319-323.

Dohrmann, K., Weisz, N., Schlee, W., Hartmann, T., \& Elbert, T. (2007). Neurofeedback for treating tinnitus. Progress in Brain 
Research, 166, 473-485, 554. https://dx.doi.org/10.1016 /s0079-6123(07)66046-4

Engelberg, M., \& Bauer, W. (1985). Transcutaneous electrical stimulation for tinnitus. Laryngoscope, 95(10), 1167-1173. https://dx.doi.org/10.1288/00005537-198510000-00003

Hiller, W., Janca, A., \& Burke, K. C. (1997). Association between tinnitus and somatoform disorders. Journal of Psychosomatic Research, 43(6), 613-624. https://dx.doi.org/10.1016/s00223999(97)00188-8

Jastreboff, P. J. (1990). Phantom auditory perception (tinnitus): Mechanisms of generation and perception. Neuroscience Research, 8(4), 221-254. https://dx.doi.org/10.1016/01680102(90)90031-9

Kahlbrock, N., \& Weisz, N. (2008). Transient reduction of tinnitus intensity is marked by concomitant reductions of delta band power. BMC Biology, 6(1), 4. https://dx.doi.org/10.1186/17417007-6-4

Kennerly, R. C. (2006). Changes in quantitative EEG and low resolution tomography following cranial electrotherapy stimulation (PhD dissertation). Retrieved from http://stress.org/wp-content/uploads/CES_Research /Kennerly Dissertation web.pdf

König, O., Schaette, R., Kempter, R., \& Gross, M. (2006). Course of hearing loss and occurrence of tinnitus. Hearing Research, 221(1-2), 59-64. https://dx.doi.org/10.1016 /j.heares.2006.07.007

Landgrebe, M., Frick, U., Hauser, S., Hajak, G., \& Langguth, B. (2009). Association of tinnitus and electromagnetic hypersensitivity: Hints for a shared pathophysiology? PloS ONE, 4(3), e5026. https://dx.doi.org/10.1371 /journal.pone.0005026

Leaver, A. M., Renier, L., Chevillet, M. A., Morgan, S., Kim, H. J., \& Rauschecker, J. P. (2010). Dysregulation of limbic and auditory networks in tinnitus. Neuron, 69(1), 33-43. https://dx.doi.org/10.1016/j.neuron.2010.12.002

Levine, R. A. (1999) Somatic modulation appears to be a fundamental attribute of tinnitus. In J. Hazell (Ed.), Proceedings of the Sixth International Tinnitus Seminar, (pp. 193-197). Cambridge, UK: British Society of Audiology.

Levine, R. A. (2004). Somatic tinnitus. In JB Snow (Ed.), Tinnitus: Theory and management (pp. 16-41). Lewiston, NY: BC Decker Inc.

Levine, R. A., Abel, M., \& Cheng, H. (2003). CNS somatosensoryauditory interactions elicit or modulate tinnitus. Experimental Brain Research, 153(4), 643-648. https://dx.doi.org/10.1007 Is00221-003-1747-3

Levine, R. A., Nam, E. C., Oron, Y., \& Melcher, J. R. (2007). Evidence for a tinnitus subgroup responsive to somatosensory based treatment modalities. Progress in Brain Research, 166, 195-207. https://dx.doi.org/10.1016/s00796123(07)66017-8

Mercola, J. M., \& Kirsch, D. L. (1995). The basis for microcurrent electrical therapy in conventional medical practice. Journal of Advancement in Medicine, 8(2), 107-120.
McMakin, C. (1998). Microcurrent treatment of myofascial pain in the head, neck, and face. Topics in Clinical Chiropractic, 5(1), 29-35.

Møller, A. R. (1997). Similarities between chronic pain and tinnitus, American Journal of Otology, 18(5), 577-585. https://dx.doi.org/10.1007/978-1-60761-145-5_14

Mühlau, M., Rauschecker, J. P., Oestreicher, E., Gaser, C., Röttinger, M., Wohlschläger, A. M., ... Sander, D. (2005). Structural brain changes in tinnitus. Cerebral Cortex, 16(9), 1283-1288. https://dx.doi.org/10.1093/cercor/bhj070

Nakai, K., Saitoh, H., Kanzaki, S., Goto, F., Tazoe, M., Kataoka, C., ... Uchiyama, K. (2008). Intractable tinnitus with deafness treated with autogenic training. Japanese Journal of Autogenic Therapy, 28(2), 56-61.

Saunders, J. C. (2007). The role of central nervous system plasticity in tinnitus. Journal of Communication Disorders, 40(4), 313-334. $\quad$ https://dx.doi.org/10.1016 /j.jcomdis.2007.03.006

Simmons, R. Dambra, C., Lobarinas, E., Stocking, C., \& Salvi, R. (2008). Head, neck, and eye movements that modulate tinnitus. Seminars in Hearing, 29(4), 361-370. https://dx.doi.org/10.1055/s-0028-1095895

Sindhusake, D., Golding, M., Wigney, D., Newall, P., Jakobson, K., \& Mitchell, P. (2004). Factors predicting severity of tinnitus: A population-based assessment. Journal of the American Academy of Audiology, 15(4), 269-280.

Van de Heyning, P., Meeus, O., Blaivie, C., Vermeire, K., Boudewyns, A., \& De Ridder, D. (2007). Tinnitus: A multidisciplinary clinical approach, $B$-ENT, 3(Suppl. 7), 3-10.

Vernon, J. (1987). Use of electricity to suppress tinnitus. Seminars in Hearing, 8(1), 29-48. https://dx.doi.org/10.1055 /s-0028-1089903

Weiler, E. W. J., Brill, K., Tachiki, K. H., \& Schneider, D. (2002). Neurofeedback and quantitative electroencephalography. International Tinnitus Journal, 8(2), 87-93.

Weisz, N., Moratti, S., Meinzer, M., Dohrmann, K., \& Elbert, T. (2005). Tinnitus perception and distress is related to abnormal spontaneous brain activity as measured by magnetoencephalography. PloS Medicine, 2(6), e153. https://dx.doi.org/10.1371/journal.pmed.0020153

Wright, E. F., \& Bifano, S. L. (1997). Tinnitus improvement through TMD therapy. Journal of American Dental Association, 128(10), 1424-1432. https://dx.doi.org/10.14219 /jada.archive.1997.0062

Yunus, M. B. (2009). Central sensitivity syndromes: An overview. Journal of Musculoskeletal Pain, 17(4), 400-408. https://dx.doi.org/10.3109/10582450903284752

Received: September 23, 2016

Accepted: November 18, 2016

Published: December 8, 2016 